\title{
Hydrodynamic Parameters of Air-Bubble Stimulated Underwater Spark Discharges
}

\author{
Y. Sun, I. V. Timoshkin, M. J. Given, M. P. Wilson, \\ T. Wang, S. J. MacGregor \\ Department of Electronic and Electrical Engineering \\ University of Strathclyde, Glasgow, United Kingdom \\ ying.sun@strath.ac.uk, igor.timoshkin@strath.ac.uk
}

\author{
N. Bonifaci \\ G2E Laboratory, CNRS \\ Grenoble, France \\ nelly.bonifaci@g2elab.grenoble-inp.fr
}

\begin{abstract}
Considerable amount of research work has been focused on investigation and optimization of strong acoustic waves generated by spark discharges in water. There are several methods to achieve and to stimulate underwater spark breakdowns, including free-discharges, wireguided and gas-bubble stimulated discharges. In the present work, air bubbles are injected into water in order to achieve spark discharges in relatively long inter-electrode gaps. This paper reports on hydrodynamic and acoustic characteristics of spark discharges stimulated by air bubbles and presents the functional relationships between the hydrodynamic and electrical parameters of such discharges, including breakdown voltage, spark plasma resistance and energy available in the discharge. A hydrodynamic analytical model has been developed and used to calculate the acoustic efficiency of the underwater spark discharges.
\end{abstract}

Keywords-Air-bubble triggered spark discharge in water, acoustic impulses, hydrodynamic parameters.

\section{INTRODUCTION}

Underwater spark plasma discharges generate strong acoustic impulses that can be used in several practical applications, for example in: material recycling and mineral processing; water treatment and sonar technology for sea bed profiling [1-3]. It is known that an increase in the interelectrode distance may result in the generation of stronger acoustic impulses, $[4,5,6]$. However, for the free-breakdown of an un-triggered water gap, any increase in the interelectrode distance requires an increase in the applied voltage, which may be impractical in terms of the insulation coordination of the acoustic pulse generation system. In [5] the free-breakdown of the un-triggered water gaps has been studied using tap water. The maximum gap length was $15 \mathrm{~mm}$ and the maximum applied voltage was $35 \mathrm{kV}$. It was found that for longer gaps, it was not possible to achieve stable spark discharges as ionic conduction was causing a significant reduction in the gap voltage over the longer time required to initiate a discharge. In [7], the use of a thin conductive wire between the electrodes allowed the distance between the electrodes to be increased, while keeping the charging voltage at a practical level. However, this approach requires the replacement of the wire after each shot, which results in a single shot/low frequency pulse operation regime. To increase the frequency of operation of the underwater spark discharge system and to minimize pre-breakdown ionic losses, gas (air) bubbles can be injected into the inter-electrode gap. Due to lower breakdown strength of air (as compared with water) and the electric field re-distribution and enhancement within these air-bubbles (due to permittivity mismatch between water and air), gas breakdown within these air-bubbles occurs, triggering the onset of streamer breakdown in the liquid. This results in the formation of the required plasma channel between the electrodes. A complete spark discharge can therefore be formed in larger inter-electrode gaps in water. If the air-bubble injection system can be incorporated into underwater spark breakdown electrode topologies, the use of air-bubbles may provide a practical solution for consistently triggering spark discharges in longer underwater gaps or gaps filled with water with higher conductivity.

The present paper investigates the functional dependency of the hydrodynamic and electrical parameters of air-bubblestimulated discharges in water. The time-dependence of the radius of the vapour/gas cavity formed by the spark discharge between electrodes, the peak magnitude of the acoustic shock generated by this cavity, the dynamic resistance of the plasma channel and the acoustic efficiency of the plasma discharges have been obtained as functions of the electrical parameters (breakdown voltage, energy available to the discharges) and the inter-electrode distance. The obtained functional dependencies of the hydrodynamic and electrical parameters of underwater discharges can be used for optimisation of plasmaacoustic systems, and for tailoring of the acoustic emission parameters for specific practical applications.

\section{EXPERIMENTAL SET-UP}

The underwater spark breakdowns were initiated by applying high voltage impulses to a HV rod electrode with a sharpened (conical in shape) end which was located above the grounded electrode in a water tank with dimensions of $2 \mathrm{~m}$ (length) $\times 1 \mathrm{~m}$ (width) $\times 1 \mathrm{~m}$ (height) filled with tap water. Both electrodes were made stainless steel and were placed in the center of the water tank. Atmospheric air was pumped into a hole in the grounded electrode, forming air-bubbles in the inter-electrode gap. The high voltage pulsed power system used in the present study was similar to that used in [5] where HV impulses were generated using a single stage capacitivebased pulsed power topology. The HV rod electrode with a $5 \mathrm{~mm}$ radius had the cone base and a tip radius of $\sim 0.8 \mathrm{~mm}$ to enhance the electrical field in its vicinity, the grounded electrode was cylindrical in shape with a $15 \mathrm{~mm}$ diameter. 
As mentioned, the air-bubbles were introduced into the spark gap from the lower two-piece-structure ground electrode. Vertically, a hole was drilled through, with an output diameter of $1 \mathrm{~mm}$ and another hole was drilled horizontally to form a "L" shaped duct within the electrode, Fig. 1. The shorter arm of the duct was connected to an aquarium pump through $6 \mathrm{~mm}$ nylon tubing and when the pump operated air-bubbles would therefore enter the vertical inter-electrode gap moving towards the HV conical electrode (not shown in Fig.1). The air flow from the pump was controlled by two serially-connected gas regulators. The pump was kept working during the $\mathrm{HV}$ tests to generate consistent and stable flow of air-bubbles.

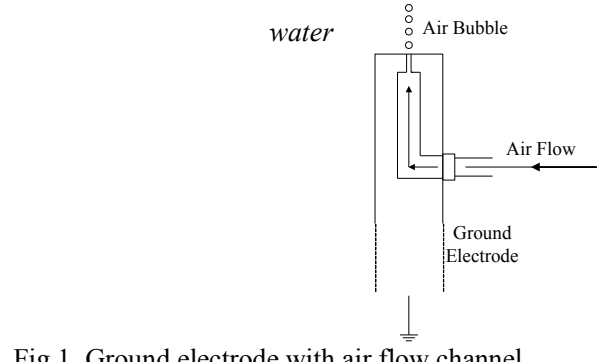

The electrical diagnostic system consisted of a PVM-5 high voltage probe (North Star Ltd USA, $80 \mathrm{MHz}$ bandwidth, 1:1000 division ratio) and a current shunt (Samtech Ltd, Scotland, 29.6 V/kA). The voltage and current signals were registered by a Tektronix TDS 2024 digitising oscilloscope (500 MHz bandwidth, $2 \mathrm{GS} / \mathrm{sec}$ sampling rate). The acoustic signals emitted into water by the gas/vapour cavity were obtained using a piezo-electric acoustic sensor, Pinducer VP1093 (Valpey Fisher Ltd USA) and were recorded by a Tektronix 5034B digitising oscilloscope (350 MHz bandwidth, $5 \mathrm{GS} / \mathrm{sec}$ sampling rate). Due to the unavailability of calibration of the acoustic sensor from the manufacturer, the output voltage from this sensor was used as a relative measure of the magnitude of the acoustic signal.

\section{RESULTS}

To investigate the acoustic and electro-hydraulic characteristics of the underwater spark discharged stimulated with injected air bubbles, different inter-electrode distances, charging voltages and capacitances of the pulsed power system were used. Three gap distances were tested: $15 \mathrm{~mm}$; $20 \mathrm{~mm}$ and $25 \mathrm{~mm}$. Three values of capacitance: $\mathrm{C}=155 \mathrm{nF}$; $266 \mathrm{nF}$ and $533 \mathrm{nF}$ were used, which were charged to 3 voltage levels: $25 \mathrm{kV} ; 30 \mathrm{kV}$ and $35 \mathrm{kV}$. However, due to the conductivity of the tap water, when using the $155 \mathrm{nF}$ capacitance it was possible to achieve repeatable spark breakdown events in $15 \mathrm{~mm}$ gap only.

\section{A. Hydrodynamic Parameters vs. Breakdown Voltage}

The period of the oscillation of the vapour/gas cavity formed by spark was measured as the time interval between two registered successive strong acoustic impulses. These impulses were clearly identified and their wave-forms are similar to those observed for free underwater discharges, [5]. In the ideal, no energy losses, case the vapour/gas cavity oscillates reaching its maximum diameter, then collapsing under the external hydrostatic pressure and then expanding again to the same size over a similar time interval. In the practical case however, the energy losses result in a reduction in the number of oscillations and in the period of each oscillation. In the present work, the term "the period of the cavity oscillation", $T_{\text {cav }}$, is reserved for the time interval between the first acoustic impulse emitted by the forming cavity during spark discharge and the second strong impulse emitted by the cavity at the moment of its collapse. Secondary oscillations of the cavity are not considered in this work. The period of the first cavity oscillation, $T_{c a v}$, was obtained for all 3 inter-electrode distances, 3 capacitances and 3 charging voltages. The functional behavior of $T_{c a v}$, is shown in Fig. 2. Each point on this graph represents a single breakdown event stimulated by air bubbles.

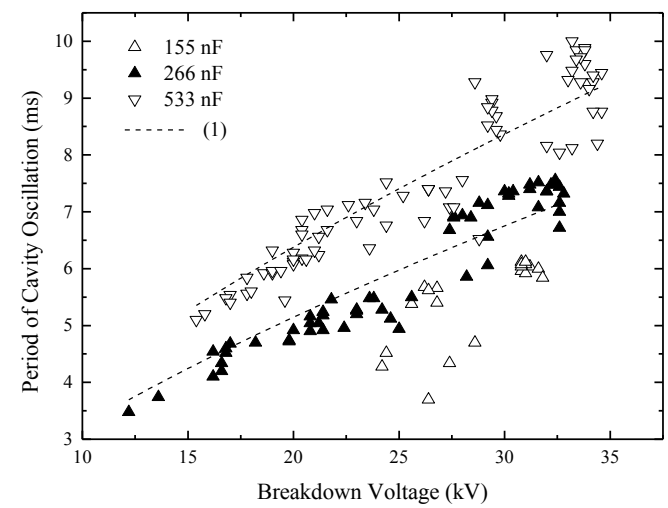

Fig.2. Period of cavity oscillation as a function of breakdown voltage. Dashed lines are fitting lines for $266 \mathrm{nF}$ and $522 \mathrm{nF}$ capacitances obtained by (1).

The dashed lines show fits to equation (1) for discharges generated using the $266 \mathrm{nF}$ and $533 \mathrm{nF}$ capacitances. No fitting for $155 \mathrm{nF}$ capacitance is provided as only a single inter-electrode distance has been used for this value of the storage capacitance. Equation (1) used in [5] for freedischarge in water links $T_{c a v}$ with the breakdown voltage, $V_{b r}$ :

$$
T_{c a v} \propto V_{b r}^{2 / 3}
$$

Fig. 2 shows that (1) provides a possible simple fitting to the experimental data and can therefore be used for the description of the functional dependency $T_{c a v}\left(V_{b r}\right)$ for bubbletriggered spark discharges. Higher $V_{b r}$ and $C$ provide higher energy available in spark discharges reflected in an increase in $T_{\text {cav }}$.

Fig. 3 shows the relationship between the peak magnitude of acoustic impulse and the breakdown voltage. The magnitude of acoustic signal increases with increasing $V_{b r}$ for all tested capacitances and inter-electrode distances. In [5] it was suggested that the functional dependency of the peak acoustic magnitude, $P_{a c}$, on the breakdown voltage, $V_{b r}$, for free discharges, was provided by (2): 


$$
P_{a c} \propto V_{b r}^{2 \theta / 3}
$$

where $\theta=1.13$ is based on the empirical behavior of underwater explosions.

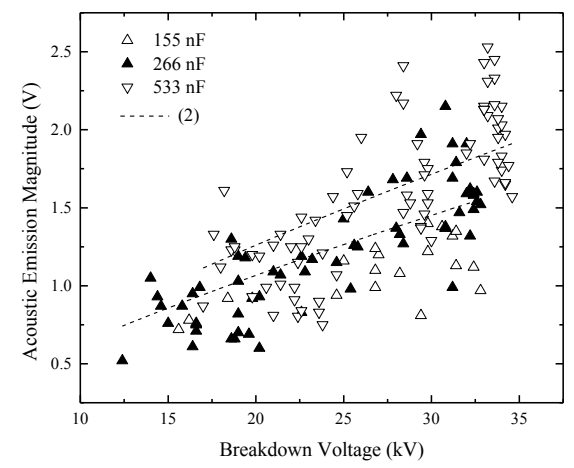

Fig. 3. Acoustic magnitude as a function of breakdown voltage. Dashed lines are fitting lines for $266 \mathrm{nF}$ and $522 \mathrm{nF}$ capacitances obtained by (2).

Assuming that the injected gas bubbles only affects $V_{b r}$ it is reasonable to suggest the same relationship (2) in the case of bubble-stimulated discharges. The dashed lines in Fig.3 provide fitting by (2) for the acoustic data obtained with $266 \mathrm{nF}$ and $533 \mathrm{nF}$ capacitances. Again, no fitting for $155 \mathrm{nF}$ capacitance is provided.

\section{B. Hydrodynamic Parameters vs Plasma Resistance}

The resistance of the plasma channel was calculated in the constant resistance approach. This approach has been verified for free discharges in water in [4] and [5]. Assuming that the post-breakdown current waveform follows the response of an underdamped $R-L-C$ circuit (3):

$$
I(t)=I_{0} \exp (-\alpha t) \sin (\omega t)
$$

where $\alpha=(2 R L)^{-1}$ and $\omega=\left((L C)^{-1}-\alpha^{2}\right)^{1 / 2}$, it is possible to obtain the total resistance, $R$, and inductance of the circuit and the plasma channel. Based on the behavior of the circuit when the gap is short circuited (no resistance of the plasma channel), the circuit resistance, $R_{\text {circuit, }}$ can also be calculated. By subtracting $R_{\text {circuit }}$ from $R$, the resistance of the postbreakdown plasma channel, $R_{p l}=R-R_{\text {circuit }}$, can be obtained. This resistance, $R_{p l}$, has been obtained for all inter-electrode gaps, charging voltages and capacitances. Fig.4 and Fig. 5 shows plots of the period of the first cavity oscillation and peak acoustic magnitude as a function of the plasma resistance, $R_{p l} . T_{c a v}$ and the peak acoustic magnitude decrease with increasing plasma resistance. The discharges with larger plasma resistances (as shown in Fig. 4 and Fig. 5) generally correspond with lower breakdown voltages at longer gap distances where less energy is available at breakdown to be transferred into the discharge channel.

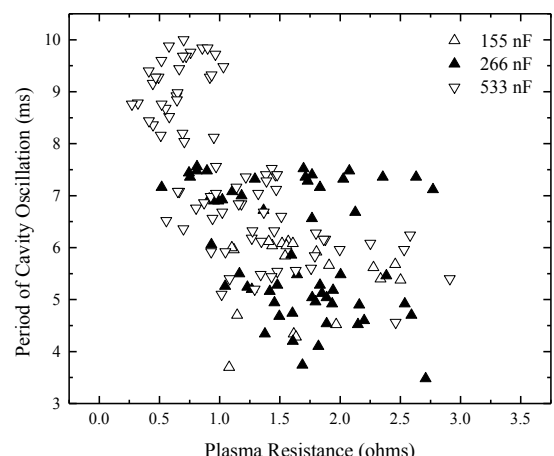

Fig. 4. Period of cavity oscillation $v s$ plasma resistance.

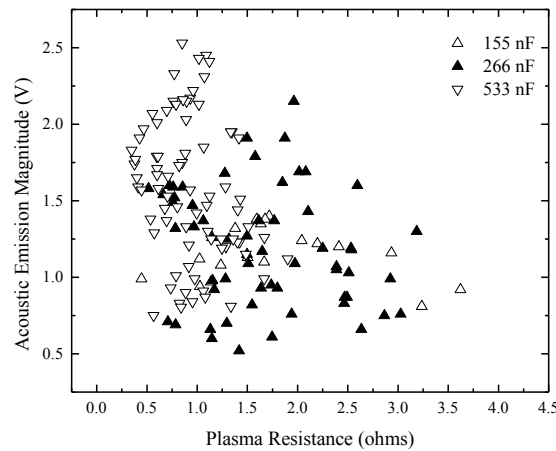

Fig. 5. Acoustic magnitudes vs plasma resistance.

Although the data in Fig. 4 and 5 show a large spread, these figures provide information on the general tendencies observed in underwater spark discharges. These graphs also demonstrate that even air-bubble triggered discharges are not stable, i.e. for the same values of plasma resistances, there will be a relatively large scattering in the period of the first oscillation and in the acoustic magnitude. This information can be important for evaluation of predictability of such discharges in practical applications.

\section{Acoustic Efficiency vs Energy}

The acoustic efficiency of the air-bubble triggered discharges is defined as the ratio of the acoustic energy in the pressure pulse and the total energy delivered into the plasma channel. The total energy, $W$, delivered into the postbreakdown plasma channel is obtained by:

$$
W=R_{p l} \int I(t)^{2} d t
$$

In the case of spherical acoustic wave radiated by the expanding cavity, its acoustic energy, $W_{a c}$, can be approximated by $[6,8]$ :

$$
W_{a c}=4 \pi Z_{a c}^{-1} a^{2} \int P_{a c}(t)^{2} d t
$$

where $P(t)$ is the acoustic pressure at the specific observation point, $a ; Z_{a c}$ is the acoustic impedance of water, defined as $Z_{a c}=\rho c$ where $\rho$ is the density of water and $c$ is the local speed of sound in water. Without knowing the exact 
calibration of the pressure sensor, the actual values of acoustic magnitude in the experimental data reported in this paper are not known and (5) cannot be used directly. Therefore, an alternative analytical model has been developed to obtain the pressure wave-forms generated by underwater spark discharges. The model is based on the Kirkwood-Bethe's gas cavity oscillation model in compressible liquid, (6), modified Tait's equation of state for water, (7), (both discussed in [6]), the energy balance equation, (8), and the acoustic pressure impulse, $P_{a c}(t)$ in water, (9), both given in [4]:

$$
\begin{gathered}
\left(1-\frac{\dot{r}}{c}\right) r \ddot{r}+\frac{3}{2}\left(1-\frac{\dot{r}}{3 c}\right) \dot{r}^{2}=\left(1+\frac{\dot{r}}{c}\right) H+\left(1-\frac{\dot{r}}{c}\right) \frac{r}{c} \dot{H} \\
H=\frac{n}{n-1} \frac{\left(\rho_{\infty}+B\right)^{\frac{1}{n}}}{\rho_{\infty}}\left[\left(P_{\ell}+B\right)^{\frac{n-1}{n}}-\left(\rho_{\infty}+B\right)^{\frac{n-1}{n}}\right] \quad c=\left(c_{\infty}^{2}+(n-1) H\right)^{\frac{1}{2}} \\
\frac{P_{\ell}+B}{P_{\infty}+B}=\left(\frac{\rho}{\rho_{\infty}}\right)^{n} \\
\left(P_{\ell}+P_{\infty}\right) \frac{d V_{c a v}}{d t}+\frac{1}{\gamma-1} \frac{d\left(P_{\ell} V_{c a v}\right.}{t}=R_{p l} I^{2} \\
P_{a c}(t)=P_{\infty}+\rho_{\infty}\left(\frac{1}{a}\left((r(t))^{2} \frac{d^{2} r(t)}{d t^{2}}+2 r(t)\left(\frac{d r(t)}{d t}\right)^{2}\right)-\frac{(r(t))^{4}}{2 a^{4}}\left(\frac{d r(t)}{d t}\right)^{2}\right)
\end{gathered}
$$

Here $r(t)$ is the time-dependent radius of the bubble, $\ddot{r}$ and $\dot{r}$ are $2^{\text {nd }}$ and $1^{\text {st }}$ time derivatives of $r(t)$ respectively; $c_{\infty}$ is the speed of sound in undisturbed water; $\rho$ and $\rho_{\infty}$ are the densities of water with and without an external pressure disturbance; $P_{\ell}$ is the pressure at the gas cavity/water interface; $V_{\text {cav }}$ is the cavity's volume; $P_{\infty}$ is the hydrostatic pressure; $H$ is the enthalpy of water at the cavity/water interface; $\gamma$ is the constant ratio of specific heats, $\gamma=1.3 ; P_{a c}(t)$ is the time-dependent pressure captured at distance $a$ from the discharge; $n$ is $\sim 7$ and $B$ is $\sim 0.3 \mathrm{GPa}$ for water, [6].

The model based on (6) - (9) was solved in MatLab R2014a. The model has been tested on sensitivity to its initial conditions. It was found that this model is not sensitive to the initial cavity radius as it expands very quickly during the energy deposition stage. It was shown that a 10-times increase in the initial cavity radius produces less than $1 \%$ change in the acoustic output. The pressure impulse emitted during the prime expansion of the post-breakdown cavity (obtained by (9)) was used in (5) to calculate the acoustic energy, $W_{a c}$; the total energy delivered to the spark channel was calculated using (4). The acoustic efficiency was defined as $\eta_{a c}=$ $W_{a c} / W$. Fig. 7 shows the relationship between, $\eta_{a c}$, and $R_{p 1}$. $\eta_{a c}$ increases with the increase of the plasma resistance for all 3 gaps, 3 capacitances and charging voltages. Comparing Fig.5 and Fig.7 it can be concluded that although the peak pressure magnitude decreases with an increase in the plasma resistance, the efficiency of transformation of the electrical energy delivered into the breakdown channel into the acoustic energy is higher for the larger values of $R_{p l}$. However this is occurring when the values of both $W_{a c}$ and $W$ are considerably smaller than those for lower plasma resistances.

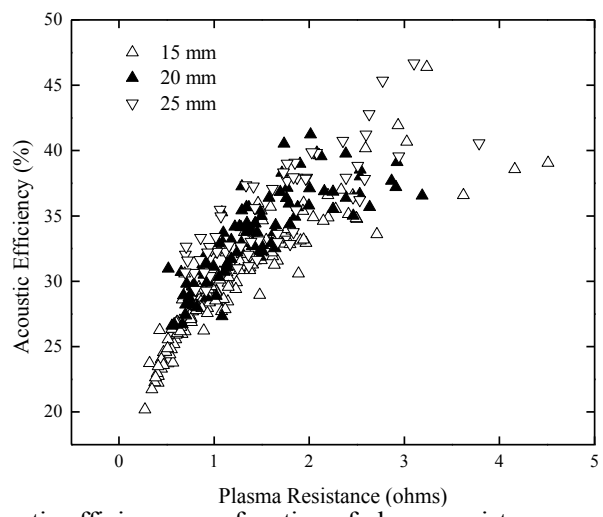

Fig.6. Acoustic efficiency as a function of plasma resistance.

\section{CONCLUSION}

The present paper reports on the hydrodynamic and acoustic characteristics of underwater spark discharges triggered with an air bubble injected into the inter-electrode gap. It was shown that this mechanism of triggering the spark discharges allows the use of increased inter-electrode distances which is not possible in the case of free, selftriggering discharges using similar electrical parameters (applied voltage and capacitance of the energy storage system). It was shown that for a given plasma resistance, the spark discharges with an increased inter-electrode length results in a higher efficiency of transformation of the electrical energy delivered into the spark post-breakdown channel into the acoustic energy radiated by the channel. Thus, this triggering method can be used to achieve an improved acoustic efficiency in the underwater spark plasma sources which will help to optimise their practical applications.

\section{REFERENCE}

[1] K. Chung, S. Lee, Y. Hwang, C. Kim, "Modeling of pulsed spark discharge in water and its applications to well cleaning", Curr. Appl. Phys., vol. 15, pp. 977-986, 2015.

[2] M. Wilson, L. Balmer, M. Given, S. MacGregor, I. Timoshkin, “An investigation of spark discharge parameters for material processing with high power ultrasound", Min. Eng., vol. 20, pp. 1159-1169, 2007.

[3] http://woodshole.er.usgs.gov/operations/sfmapping/sparker.htm.

[4] I. Timoshkin, R. Fouracre, M. Given, S. MacGregor, "Hydarodynamic modeling of transient cavities in fluids generated by high voltage spark discharges", J. Phys. D: Appl. Phys., vol. 39, pp. 4808-4817, 2006.

[5] Y. Sun, I. Timoshkin, M. Given, et al, "Impulsive discharges in water: acoustic and hydrodynamic parameters", IEEE Trans. on Plasma Sci., vol. 44, pp. 2156-2166, 2016.

[6] R. M. Roberts, J. A. Cook, R. L. Rogers, "The energy partition of underwater sparks", J. Acoust. Soc. Amer., vol. 99, no. 6, pp. 34653475,1996

[7] I. Timoshkin, S. MacGregor, M. Given, R. Fouracre, "High power ultrasound impulses induced by wire-guided spark discharges in water," IEEE Int. Pulsed Power Conf., Albuquerque, pp. 33-36, 2007.

[8] S. Buogo, J. Plocek, K. Vokurka, "Efficiency of energy conversion in underwater spark discahrges and associated bubble oscillations: experimental results", ACTA, vol. 95 46-59, pp. 46-59, 2009. 